\title{
El estrés laboral y su incidencia en el desempeño del personal administrativo
}

\section{Work stress and its impact on the performance of administrative staff}

\author{
Claudio Eduardo Soriano Tumbaco ${ }^{1}$ \\ ${ }^{1}$ Universidad Estatal Península de Santa Elena, Ecuador, Ecuador \\ *1.alo26@hotmail.es
}

DOI: https://doi.org/10.26871/killkanasocial.v5i1.735

\begin{abstract}
Resumen
Los recursos humanos son parte de los componentes más importantes de los procesos dentro de una organización. En la UPSE, existe un estudio socioeconómico del trabajador, desarrollado por la Unidad Administrativa de Talento Humano, pero en la actualidad no existe una caracterización de sus grupos profesionales, según los riesgos psicosociales, pues su estudio se encuentra en desarrollo. El objetivo de este trabajo es contribuir al desarrollo de los perfiles de riesgos psicosociales y de salud, desde la perspectiva del desempeño laboral. Se tomó el cuestionario Maslach Burnout Inventory (MBI) como instrumento principal para medir el estrés laboral del personal administrativo. Su función es medir el desgaste profesional, así como la frecuencia y la intensidad con la que se sufre el Burnout. Este test es conocido como el test de Burnout y está estandarizado para ámbitos laborales. En este estudio se buscó medir la confiabilidad de las respuestas ofrecidas, para lo que se utilizó el Coeficiente Alfa de Cronbach. Se recibieron respuestas de 78 encuestados, que significan el 40,6\% de la población total. Las respuestas no alcanzaron el tamaño de la muestra, aun cuando el cuestionario fue enviado en tres ocasiones. Los resultados obtenidos en esta investigación permiten afirmar que no existe presencia de estrés laboral en el personal administrativo de la Universidad Estatal Península de Santa Elena. Al analizar las dimensiones del Síndrome de Burnout en la muestra estudiada se confirma que existe un nivel bajo de indicios de Cansancio emocional y de despersonalización.
\end{abstract}

Palabras clave: talento humano, estrés laboral, desempeño laboral.

\begin{abstract}
Human resources are part of the most important components of the processes within an organization. In the UPSE, there is a socioeconomic study of the worker, developed by the Administrative Unit of Human Talent, but at present there is no characterization of their professional groups, according to psychosocial risks, since their study is under development. The objective of this work is to contribute to the development of health and psychosocial risk profiles, from the perspective of job performance. The Maslach Burnout Inventory (MBI) questionnaire was used as the main instrument to measure work stress in administrative personnel. Its function is to measure professional burnout, as well as the frequency and intensity with which burnout is suffered. This test is known as the Burnout test and is standardized for work environments. In this study we sought to measure the reliability of the answers offered, for which the Cronbach's Alpha Coefficient was used. Responses were received from 78 respondents, representing $40.6 \%$ of the total population. The responses did not reach the sample size, even though the questionnaire was sent three times. The results obtained in this research allow us to affirm that there is no presence of work stress in the administrative staff of the Santa Elena Peninsula State University. When analyzing the dimensions of the Burnout Syndrome in the studied sample, it is confirmed that there is a low level of signs of emotional fatigue and depersonalization.
\end{abstract}

Keywords: human talent, job stress, job performance.

\section{Introducción}

Los recursos humanos son parte de los componentes más importante de los procesos dentro de una organización sea esta empresarial o no. Su importancia está dada en el valor que encierra la formación de cada uno y el sentido humano de su participación. El mismo está respaldado, en el plano social, por la diversidad de caracteres, culturas, nacionalidades, y géneros. En el plano individual, se muestra muy plural debido a las características de la personalidad, del temperamento, y del resto de los procesos psíquicos y representaciones individuales que conforman la subjetividad única e irrepetible del ser humano. Todos 
estos elementos hacen que se complejice el proceso de estandarización de exigencias, comportamientos laborales frente a las circunstancias cotidianas en la ejecución de sus funciones de trabajo. Son múltiples los estudios desarrollados por instituciones científicas y las propias organizaciones productivas, que demuestran la importancia del ser humano en todos sus procesos. Las mismas han estimulado una fuente inagotable de acciones para mejorar los factores que afectan a las personas, y así perfeccionar el entorno de trabajo y obtener mayor eficiencia y productividad.

Los riesgos psicosociales según Rick et al (2001) abarcan un crisol de factores como la carga de trabajo, el contenido mismo, los tipos de supervisión y control, el grado de libertad para la realización de la actividad, la evaluación de su desempeño, entre otros, que permiten hacer diagnosticar los elementos de riesgo psicosocial a los que se encuentra sometido el trabajador, siendo el Síndrome de Bournot o "Síndrome del quemado", una de las consecuencias a las que conducen estos riesgos.

Según Salgado y Lería (2020), desde el concepto primario establecido por el modelo de Maslach, el Burnout se ha entendido como una conflicto evidente para el contrarrestar el estrés a consecuencia de las características del trabajo. Comprende la presencia de sintomatología como decaimiento, fatiga física y emocional, irritabilidad y depresión, se manifiesta en personas sin psicopatología anteriores y causa un desempeño laboral deficiente debido a actitudes y disposiciones negativas al trabajo.

En la UPSE, existe un estudio socioeconómico del trabajador, desarrollado por la Unidad Administrativa de Talento Humano, pero en la actualidad no existe una caracterización de sus grupos profesionales, según los riesgos psicosociales, pues su estudio se encuentra en desarrollo. La Unidad de Seguridad y Salud del Trabajador es de reciente creación en la institución, razón por la cual se están estudiando aún los perfiles de riesgos psicosociales y de salud. En este sentido, esta investigación puede contribuir al desarrollo de los mismos, desde la perspectiva del desempeño laboral, con independencia de que según Greig \& Fuentes,(2009), existen múltiples variables como la edad, el estado civil, el trabajo en turnos que exceden 12 y 24 horas o el tiempo de permanencia en el puesto, que también han demostrado relación causal con el mencionado síndrome de Bournot.

El empeño de la voluntad de modo intenso, con sobrecarga y sostenido en el tiempo, por tratar de adaptarse y responder a un exceso en las demandas y presiones laborales, originan riesgos y síntomas que afectan la salud y por lo tanto el rendimiento laboral y el servicio que se debe prestar, según Maslach et al., (1986) este síndrome es un tipo específico de stress laboral, que parte del desgaste profesional.

Existen componentes personales que según Ayala y Stortti, (2006) provocan una profundización del estrés laboral, como componentes personales pre mórbidos que incrementan la tendencia hacia la baja de defensas in- munológicas, incrementan la susceptibilidad hacia factores ambientales y otros sociales, que perjudican el desempeño.

El Síndrome de Burnout es considerado ampliamente en las actividades vinculadas a la actividad humana, especialmente en las académicas y laborales en general, pues su presencia está determinando el nivel de agotamiento y desgaste de las personas, lo que puede llegar a ser considerado como estrés. El Burnout es considerado un riesgo laboral por la Organización Mundial de la Salud. Existen técnicas específicas y cuestionarios estandarizados, para medir la presencia de este síndrome en las personas.

Maslach, y sus colaboradores (1986) fueron los primeros en definir al Burnout como "un síndrome de agotamiento emocional, despersonalización y baja realización personal, que puede ocurrir en individuos que trabajan con personas"(p.404)

Al reflexionar la amplitud de esos tres aspectos psicológicos: la emoción, la personalidad y la autoestima, es que esta investigación asume este concepto como base, no obstante en la ciencia psicológica permanecen muchas teorías que retoman y establecen otros aspectos, a tener en cuenta también, pero en esencia consideraremos el concepto primigenio de Maslach.

El Síndrome del Quemado más tarde Freudenberger (1974) lo conceptualiza como una autopercepción de fracaso y un existir agotado o gastado, como resultante de una sobrecarga por exigencias de energías y recursos personales o esfuerzo psicológico del trabajador.

Se aprecia un sin número de conceptos que abordan el Síndrome de Bournot, pero todos coinciden que en ellos se evidencia una progresiva pérdida del interés y emoción hacia las personas de su entorno laboral, ya sean estos clientes internos o externos.

Esto es precisamente lo que obstaculiza el diagnóstico del Síndrome del Quemado, la carencia de una definición universal, pero múltiples investigadores concuerdan en que se trata de un estado de estrés laboral crónico que termina en el menoscabo de la sistematización del rol y del rechazo al puesto de trabajo (López y Bernal, 2002; Martínez, Vilaregut, y Roig, 2004; Ortega y López, 2004; Ferrer, Solano, Vizcaya, y Vidal, 2002).

Tampoco existe una caracterización del personal administrativo proclive a desarrollar el síndrome del desgaste profesional. Variables como el trabajo en turnos que exceden las 8 horas, la edad cronológica, el estado civil, o la antigüedad profesional han demostrado relación causal con el mencionado síndrome, en personal administrativo de centros de salud. (Greig y Fuentes, 2009).

Plantean Salgado y Lería (2020) que "a escasez de evidencia empírica actualizada hace de su indagación un aspecto relevante a considerar en la exploración de las características que adquiere el Síndrome de Burnout, en estos colectivos laborales y contextos socioeconómicos que les son propios, especialmente en las áreas administrativas" pág.8. 
El Síndrome de Bournot es un proceso multicausal y muy complejo, donde además de las ya descritas anteriormente se plantean otras causas, entre las que más figuran: falta de estimulación sobrecarga de trabajo, , aburrimiento, stress, aislamiento, escasa orientación profesional, crisis en el desarrollo profesional, limitadas condiciones económicas, horarios inapropiados, ausencia de recursos humanos en el mismo turno, entre otros. Ayala, (2006) refiere que a todo ello colaboran también, componentes personales pre mórbidos que aumentan la susceptibilidad personal, inadecuada formación profesional, En el estudio de Cázares y Moral, (2016) se describen factores laborales o profesionales, factores sociales, y factores ambientales, así como formaciones psicológicas: rasgos de personalidad, neurosis, introversión, y mayor tendencia a responder en sentido socialmente deseable para dar una imagen social positiva al ser encuestado o entrevistado, por lo que muchas veces estos resultados no predicen niveles de estrés, sino que por la estructura del cuestionario facilitan información real y no predictiva.

Teniendo en cuenta los potenciales, según Navarro y Climent (2017) así como los factores de inseguridad inherentes a la ordenación laboral expuestos hasta ahora, y sobre todo la insuficiencia de trabajos donde se aborden variables de actividad psíquica y social personal en colectivos administrativos, se desarrolla este estudio a nivel descriptivo y exploratorio.

El objetivo general de esta investigación es analizar la posible presencia del estrés laboral en el desempeño del personal administrativo de la Universidad Estatal Península de Santa Elena, para lo cual se plantearon como objetivos específicos el análisis del nivel de estrés laboral, mediante el test de Bournot, así como la medición de su desempeño laboral, mediante los instrumentos que se utilizan habitualmente para fines de evaluación, según la normativa. Se realizaron procedimientos estadísticos que permitieron establecer las características de ambas variables en la muestra de estudio.

\section{Metodología}

Se tomó el cuestionario Maslach Burnout Inventory (MBI) como instrumento principal para medir el estrés laboral del personal administrativo. El mismo está constituido por 22 ítems en forma de afirmaciones, sobre los sentimientos y actitudes del profesional en su trabajo. Su función es medir el desgaste profesional, así como la frecuencia y la intensidad con la que se sufre el Burnout.

Este test es conocido como el test de Burnout y está estandarizado para ámbitos laborales. Para ambientes complejos de empresas de altas tensiones por la naturaleza de su misión, el mismo ha sido objeto de múltiples adaptaciones. La variante utilizada en esta investigación es la más difundida, al tratarse de un ambiente administrativo, sin niveles de tensión que comprometan la vida humana, etc.

Los valores referenciales de esta prueba son los siguientes:
Tabla 1: Valores referenciales

\begin{tabular}{lccc}
\hline \multirow{2}{*}{ Dimensión } & \multicolumn{3}{c}{ Niveles } \\
\cline { 2 - 4 } & BAJO & MEDIO & ALTO \\
\hline Cansancio emocional & $0-18$ & $19-26$ & $27-54$ \\
Despersonalización & $0-5$ & $6-9$ & $10-30$ \\
Realización personal & $0-33$ & $34-39$ & $40-56$ \\
\hline
\end{tabular}

En este estudio se buscó medir la confiabilidad de las respuestas ofrecidas, para lo que se utilizó el Coeficiente Alfa de Cronbach, de 0 a1 y mientras el resultado esté más cercano a 1 es más confiable, considerando lo ideal entre 0.8 y 0.99, Hernández Sampieri et al., (2010).

Para el cálculo de este coeficiente se consideró en el cálculo el número de ítems, en este caso las 22 variables del test de Burnout, la varianza de cada ítem y la varianza total (Ecuación 1).

$$
\alpha=\frac{K}{K-1} *\left[1-\frac{\sum V i}{V t}\right]
$$

Donde:

$\alpha$ : Coeficiente Alfa de Cronbach.

$\mathrm{K}$ : número de ítems.

Vi: varianza de cada ítem.

Vt: varianza total.

Para su desarrollo se halló un coeficiente global y además parcial para las 3 dimensiones del test.

Se realizó también un análisis de las respuestas de cada una de las preguntas del cuestionario y de cada una de las dimensiones, donde se determinó el promedio de las respuestas, la desviación estándar y la moda. Luego las respuestas en que los estadígrafos (media y moda) tenían valores que reflejaban más frecuencia de aparición, se realizó el análisis correspondiente.

La población está constituida por 192 personas, que realizan funciones de: Analistas, asistentes administrativos, técnicos de archivo, técnicos de biblioteca, técnicos de informática, técnicos de laboratorio, técnicos docentes, entre otros.

Se realizó un muestreo probabilístico aleatorio simple con un nivel de confianza del $95 \%$ y un margen de error del $5 \%$, por lo cual el tamaño de la muestra, fue de 129. El cuestionario se elaboró usando la herramienta Google forms y se distribuyó por correo electrónico a toda la población, para responder en línea.

\section{Resultados y discusión}

Se recibieron respuestas de 78 encuestados, que significan el 40,6\% de la población total. Las respuestas no alcanzaron el tamaño de la muestra, aun cuando el cuestionario fue enviado en tres ocasiones. Esta tasa de respuesta, que podría considerarse baja, coincide con lo plateado por Sampieri et al. (2010) para cuestionarios administrados por correo electrónico o páginas web. pág. 244. 
El coeficiente Alfa de Cronbach, indicador de la validez y confiabilidad interna del instrumento, se determinó de forma global, para las 22 variables del Test de Burnout, obteniendo un valor de 0,75 que permite calificar la confiabilidad como Buena, según Oviedo y Campo (2005).
Bojórquez et al. (2013) plantean que valores superiores a 0,7 demuestran que la relación entre variables es fuerte, mientras que si el valor es inferior y cercano a cero refleja que la relación entre ellas es débil.

Tabla 2: Resultados del Test de Burnout

\begin{tabular}{|c|c|c|c|c|c|c|}
\hline No. & Ítems & Promedio & $\begin{array}{l}\text { Deviación } \\
\text { Estándar }\end{array}$ & Moda & $\begin{array}{l}\text { Indicios de } \\
\text { Burnout }\end{array}$ & $\begin{array}{l}\text { Alfa de } \\
\text { Cronbach }\end{array}$ \\
\hline \multicolumn{7}{|c|}{ Dimensión Cansancio emocional } \\
\hline 1 & $\begin{array}{l}\text { Me siento emocionalmente agotado/a por mi } \\
\text { trabajo }\end{array}$ & 2,46 & 1,83 & 1 & \multirow{9}{*}{$14,46<26,00$} & \multirow{9}{*}{0,84} \\
\hline 2 & $\begin{array}{l}\text { Me siento cansado al final de la jornada de } \\
\text { trabajo }\end{array}$ & 3,17 & 1,93 & 5 & & \\
\hline 3 & $\begin{array}{l}\text { Cuando me levanto por la mañana y me enfrento } \\
\text { a otra jornada de trabajo me siento fatigado }\end{array}$ & 1,79 & 1,76 & 1 & & \\
\hline 6 & $\begin{array}{l}\text { Siento que trabajar todo el día con compañe- } \\
\text { ros/as supone un gran esfuerzo y me cansa }\end{array}$ & 0,69 & 1,15 & 0 & & \\
\hline 8 & $\begin{array}{l}\text { Siento que mi trabajo me está desgastando. Me } \\
\text { siento quemado por mi trabajo }\end{array}$ & 1,73 & 1,90 & 1 & & \\
\hline 13 & Me siento frustrado/a en mi trabajo & 0,65 & 1,04 & 0 & & \\
\hline 14 & Creo que trabajo demasiado & 2,59 & 2,31 & 0 & & \\
\hline 16 & $\begin{array}{l}\text { Trabajar directamente con compañeros/as me } \\
\text { produce estrés }\end{array}$ & 0,71 & 1,15 & 0 & & \\
\hline 20 & $\begin{array}{l}\text { Me siento acabado en mi trabajo, al límite de } \\
\text { mis posibilidades }\end{array}$ & 0,69 & 1,27 & 0 & & \\
\hline \multicolumn{7}{|c|}{ Dimensión Despersonalización } \\
\hline 5 & $\begin{array}{l}\text { Creo que estoy tratando a mis compañeros/as } \\
\text { como si fueran objetos impersonales }\end{array}$ & 0,37 & 1,04 & 0 & \multirow{5}{*}{$15,33<6,00$} & \multirow{5}{*}{0,14} \\
\hline 10 & $\begin{array}{l}\text { Me he vuelto más insensible con la gente desde } \\
\text { que ejerzo la profesión }\end{array}$ & 0,69 & 1,62 & 0 & & \\
\hline 11 & $\begin{array}{l}\text { Pienso que este trabajo me está endureciendo } \\
\text { emocionalmente }\end{array}$ & 0,65 & 1,11 & 0 & & \\
\hline 15 & $\begin{array}{l}\text { No me preocupa realmente lo que les ocurra a } \\
\text { algunos de mis compañeros }\end{array}$ & 2,96 & 2,59 & 0 & & \\
\hline 22 & $\begin{array}{l}\text { Creo que los compañeros/as me culpan de algu- } \\
\text { nos de sus problemas }\end{array}$ & 0,66 & 1,17 & 0 & & \\
\hline \multicolumn{7}{|c|}{ Dimensión Realización personal } \\
\hline 4 & $\begin{array}{l}\text { Tengo facilidad para comprender como se sien- } \\
\text { ten mis compañeros/as }\end{array}$ & 3,89 & 1,94 & 6 & \multirow{8}{*}{$37,93>34,00$} & \multirow{8}{*}{0,72} \\
\hline 7 & $\begin{array}{l}\text { Creo que trato con mucha eficacia los proble- } \\
\text { mas de mis compañeros/as }\end{array}$ & 4,30 & 1,83 & 6 & & \\
\hline 9 & $\begin{array}{l}\text { Creo que con mi trabajo estoy influyendo posi- } \\
\text { tivamente en la vida de mis compañeros/as }\end{array}$ & 4,56 & 1,81 & 6 & & \\
\hline 12 & Me siento con mucha energía en mi trabajo & 5,12 & 1,51 & 6 & & \\
\hline 17 & $\begin{array}{l}\text { Siento que puedo crear con facilidad un clima } \\
\text { agradable con mis compañeros/as }\end{array}$ & 5,03 & 1,57 & 6 & & \\
\hline 18 & $\begin{array}{l}\text { Me siento motivado después de trabajar en con- } \\
\text { tacto con compañeros/as }\end{array}$ & 4,96 & 1,43 & 6 & & \\
\hline 19 & $\begin{array}{l}\text { Creo que consigo muchas cosas valiosas en este } \\
\text { trabajo }\end{array}$ & 5,23 & 1,36 & 6 & & \\
\hline 21 & $\begin{array}{l}\text { En mi trabajo trato los problemas emocional- } \\
\text { mente con mucha calma }\end{array}$ & 4,82 & 1,77 & 6 & & \\
\hline
\end{tabular}

Fuente: Autor 
Al analizar la dimensión de Cansancio emocional, Tabla No.2, se aprecia que en las preguntas 6, 13, 16 y 20 el promedio de las respuestas es inferior a 1, sin embargo la moda en ellas, excepto en la pregunta 3 , resulta 0 . Lo que evidencia que para la mayoría de los encuestados no existe cansancio sistemático.

Las preguntas 1,2 y 14 se relacionan entre si y las respuestas tienen como promedio el 2,45 y 3,46, o sea que manifiestan sentirse medianamente agotados, cansados desde el punto de vista emocional, lo que justifica la idea de que perciben que trabajan demasiado en sus puestos. De esta manera, hay una construcción muy subjetiva, que evidencia una percepción de equilibrio entre las tareas que desempeñan. En este caso la desviación estándar dice que las respuestas han sido de una gran diversidad, por lo que en esta dimensión de Cansancio emocional, el análisis se centra en considerar el valor de la moda, que está en el rango entre 0 y 1 , excepto el Item 2 cuya moda es 5 . Este valor ofrece consistencia a los datos y permite rechazar la idea de que existe alto nivel de cansancio.

Los resultados de esta encuesta coinciden con los de una encuesta aplicada de forma exploratoria al personal administrativo, dos meses antes de aplicar el Test de Burnout, según se aprecia en la figura No.1.

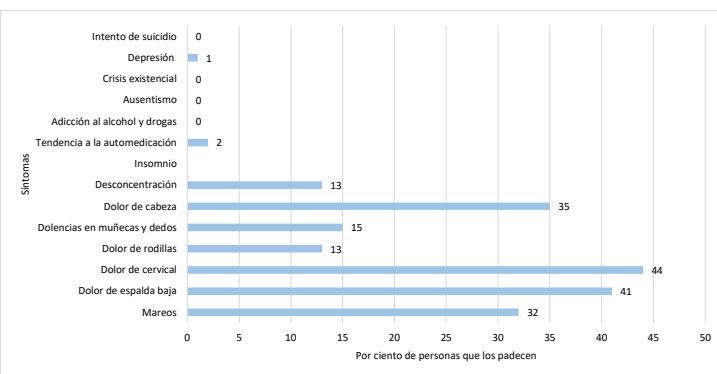

Figura 1: Síntomas que presentan administrativos UPSE Elaboración: Autor

Se describen síntomas menores, dolor de cervical (44\%), dolor de espalda baja (41\%) y dolor de cabeza $(35 \%)$, relacionados básicamente con la postura, provocadas en su mayoría por causas físicas, aunque según Caramés (2020), se presentan muchos de estos padecimientos, cuando los aspectos psiconeuroemocionales del sujeto se encuentran sobrecargados, tanto en sus relaciones humanas como en las laborales y también producto de elementos posturales. Este resultado se asemeja al encontrado por Marenco y Ávila (2016), refiriendo que las dificultades relacionadas con la salud mental en sus muestras estudiadas, tampoco mostraron prevalencias altas, pero resaltó la sintomatología somática, de órganos físicos con un porcentaje de $19.1 \%$. Muy pocos en su caso, al igual que la muestra UPSE, presentó ansiedad e insomnio. En el caso de este estudio el tercer indicador más frecuente fue la disfunción social cuyo reporte fue de $15.3 \%$ y el último lugar lo tuvo la depresión que solo se reportó en $0.4 \%$
En resumen el caso más crítico en esta dimensión es la respuesta al ítem que plantea me siento cansado al final de la jornada, en la que la moda se corresponde con "unas pocas veces a la semana". Este resultado se acerca al obtenido por Rivas y Barraza (?), donde las medias altas se ubican en ese mismo ítem y la cual arroja una media de 2.48, frente a una de 3.17 en la encuesta UPSE, lo que explica que si su moda es de 5, este fenómeno no ocurre siempre, sino unas pocas veces a la semana, pero ciertamente sucede.

En otro orden de análisis, profundizando lo anterior, en este $46 \%$ de los encuestados que expresa sentir cansancio al final de la jornada, se encuentran mayoritariamente hombres entre los 41 y 60 años con diversas ocupaciones en el ámbito administrativo. El grupo ocupacional no fue significativo en los resultados masculinos, mientras que en las mujeres de ese mismo rango de edades, expresaban cansancio, en mayor medida las asistentes administrativas.

Este elemento específicamente guarda relación desde el punto de vista psicológico con la apreciación que evidencia la dimensión Despersonalización, en la que su indicio de Burnout es bajo, ver tabla No.1, aunque resulta significativo el promedio de respuesta al ítem 15 , que refiere no preocupar realmente lo que ocurra a sus compañeros de trabajo, con una media de 2,96 y una desviación estándar de 2,59, sin embargo la moda es 0 . Esto induce la idea de que es en muy pocos trabajadores los que consideran mínimamente no importarle lo que les ocurra a sus colegas.

Esta cifra se encuentra por debajo del resto de ítems de esta dimensión de Despersonalización, donde los demás apuntan a relaciones cordiales, de respeto y compromiso entre los trabajadores administrativos, por lo que en puntuación general evidencia un índice inferior a 6, indicando que se considera un nivel muy bajo de Despersonalización. Contrastan estos datos con las investigaciones de Bustamante et al (2016), en que sus indicios de Bournot, alcanzaron el $46 \%$ de los encuestados en una institución de Carabobo, considerándose un nivel alto. Por otro lado, Salgado y Leria (2020) también encuentran niveles altos de Despersonalización en su muestra al alcanzar un 11,47 de media entre la puntuación de sus encuestados, y ellos le correlacionan de manera positiva, con las excelentes condiciones de trabajo creadas para la adecuada interrelación entre las personas del ámbito administrativo de la institución investigada.

En referencia a la dimensión de Realización personal si se analizan los ítems relacionados con el se aprecia en la tabla No. 1, que los resultados se encuentran en un nivel medio, pues la media se encuentra oscila entre 3,90 en el ítem Tengo facilidad para comprender como se sienten mis compañeros/as y 5.28. en el que plantean que Creo que consigo muchas cosas valiosas con este trabajo, lo que expresa un nivel de satisfacción muy elevado.

Sin embargo, al analizar la sumatoria de los promedios de las preguntas que incluyen la dimensión de Realización personal, se aprecia un nivel medio del Síndrome de Bur- 
nout, pues su resultado final es de 37,93 al considerar que los valores referenciales de este nivel medio oscilan entre 34 y 39.

En este sentido, existen estudios que valoran la presencia de la dimensión psicológica de Realización personal en la profesión: Pozos, Torres, Aguilera, Acosta \& González, (2008); Pozos et al., (2011), La Porta, (2010); Rolander, Stenstrom \& Jonker, (2008) Divaris, Lai, Polychronopoulou, Eliades \& Katsaros, (2012); Cázares y Moral (2016). Estos autores evalúan su frecuencia de aparición y describen como en muchos casos es escasa la realización personal, y coinciden en asociarla a la falta de propensión a la empatía y contagio emocional positivo que existe en las áreas administrativas de las organizaciones.

Estos elementos que están formalizando de cierto modo, el nivel de estrés o de agotamiento emocional en la vida laboral, van a determinar el desempeño. Los factores que implican riesgo psicosocial obtenidos en este estudio, no son consistentes con los resultados encontrados en otros estudios realizados en trabajadores universitarios, Gillespie (2001), arroja que las principales fuentes que afectaron al trabajador y a su desempeño laboral fueron: sobrecarga de trabajo, pobre desempeño gerencial, e inseguridad en el trabajo. Singh (2015), señala que sus hallazgos estuvieron centrados en excesivas demandas de trabajo, la falta de control sobre el trabajo, inadecuada definición de competencias, malas condiciones de trabajo y de recursos, y también en el estudio realizado por Unda (2016), se reconocen factores comunes como la sobrecarga de trabajo, la falta de recursos, las limitaciones presupuestarias y en general la precariedad para el desempeño de las funciones.

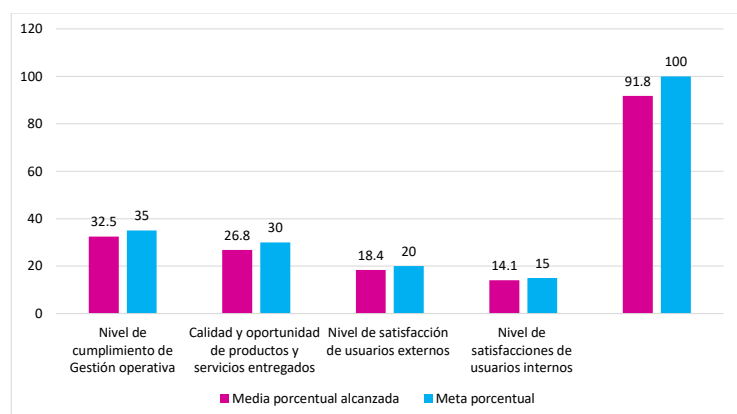

Figura 2: Resumen de indicadores cuantitativos de evaluación del desempeño personal administrativo

Elaboración: Autor

Uno de los hallazgos importantes a considerar en esta investigación radica en que como se aprecia en la Figura 2, sobre los resultados del desempeño a partir de indicadores cuantitativos en el mismo personal administrativo, el nivel de cumplimiento en cada uno de ellos es bastante cercano a las metas señaladas en el instrumento de evaluación establecido.

Se aprecia que los trabajadores administrativos de la UPSE alcanzan un $91 \%$ de las metas trazadas en sentido general. El indicador de calidad y oportunidad de productos y servicios entregados, es el que más difiere de la meta con 3,2 puntos porcentuales por debajo, pero aun así muestra un comportamiento muy positivo.

En relación a los indicadores cualitativos del desempeño, se aprecia en la figura 3 que los mismos también se manifiestan de forma efectiva en el personal administrativo estudiado.

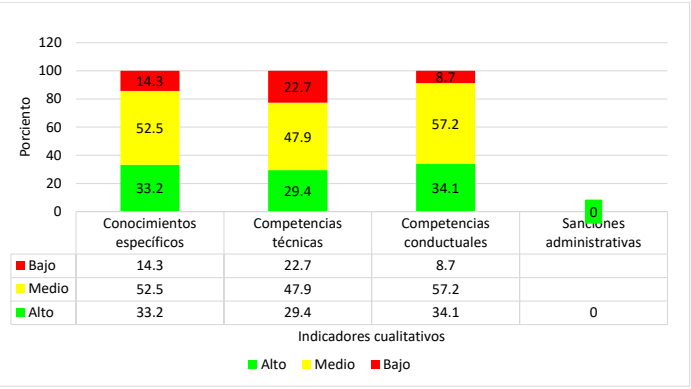

Figura 3: Resumen de resultados del desempeño laboral de trabajadores administrativos de la UPSE

Elaboración: Autor

En todos los casos, es decir en conocimientos específicos, competencias técnicas y conductuales la mayoría de los trabajadores se encuentran evaluados en niveles medios y altos. Solo pequeños grupos son calificados en un nivel bajo de estos indicadores. La expresión de sus competencias desarrolladas, se expresa en que en los últimos periodos, no existen sanciones administrativas aplicadas a este personal.

\section{Conclusiones y recomendaciones}

Los resultados obtenidos en esta investigación permiten afirmar que no existe presencia de estrés laboral en el personal administrativo de la Universidad Estatal Península de Santa Elena. Al analizar las dimensiones del Síndrome de Burnout en la muestra estudiada se confirma que existe un nivel bajo de indicios de Cansancio emocional y de despersonalización. Al valorar la dimensión de Realización personal, arroja un nivel medio de indicio de Burnout, el cual es psicológicamente insignificante, dado que la mayoría, mediante la calidad de la respuesta contenida en la moda correspondiente a los ítems que integran la dimensión, se acerca a un nivel de reconocimiento de su realización personal con la mayor frecuencia posible.

Esta situación favorable, permite explicar los resultados finales de la evaluación del desempeño de los trabajadores administrativos de la UPSE, donde existe un predominio del criterio evaluativo considerado como muy bueno al superar el $91 \%$ de los indicadores cuantitativos con resultados positivos. En el caso de los indicadores cualitativos, destacan las competencias conductuales con una mejor valoración, por encima de los conocimientos específicos y competencias técnicas. No existe ninguna sanción administrativa.

Aun cuando la situación es favorable en ambas variables, es decir tanto en el estrés como en el desempeño laboral, se realizan las siguientes recomendaciones: 
Actualizar el modelo gerencial de algunos departamentos, donde se realice una mayor cantidad de trabajo en equipo y colaborativo por las posibilidades de integración y de realización personal que promueve el mismo.

Analizar y reforzar la descripción de cargos y funciones desempeñados por los trabajadores, donde se establezcan a nivel departamental mecanismos más actualizados para la planificación, la ejecución y el control del trabajo, de forma que mediante un sistema de gestión del desempeño, se facilite la retroalimentación del personal, sobre el cumplimiento de metas fijadas y su participación en el mismo, así como hacer sentir a los trabajadores satisfechos con su trabajo, generándole una percepción adecuada de su salud, como forma de fortalecer la dimensión de la Realización personal.

Orientar parte del presupuesto de la institución al mantenimiento y mejoramiento de su infraestructura, donde el mobiliario, la iluminación y los recesos, encaucen el comportamiento ergonómico adecuado para evitar dolores de espalda y de cervical básicamente.

\section{Referencias Bibliográficas}

Bojórquez-Molina, J. A., López-Aranda, L., HernándezFlores, M. E., y Jiménez-López, E. (2013, August). Utilización del alfa de cronbach para validar la confiabilidad de un instrumento de medición de satisfacción del estudiante en el uso del software minitab del sur de sonora, cd. obregón, sonora, méxico. Cancun, Mexico..

Bustamante, E., Bustamante, F., González, G., y Bustamante, L. (2016). El burnout en la profesión docente: un estudio en la escuela de bioanálisis de la universidad de carabobo sede aragua, venezuela. Rev. Medicina y Seguridad del Trabajo, 62(243), 111-121.

Caramés, R. (2020). La biopsiconeuroemoción y los conflictos en la adultez. Cuenca. Ecuador: Editorial Tocororo.

Celina Oviedo, H., y Campo Arias, A. (2005). Aproximación al uso del coeficiente alfa de cronbach. Revista Colombiana de Psiquiatría, XXXIV(4), 572-580.

Cázares, F., y Moral, J. (2016). Diferencias sociodemográficas y de práctica profesional asociadas a estrés percibido entre dentistas colegiados de monterrey. Avances en Psicología Latinoamericana, 34(1), 8397. doi: dx.doi.org/10.12804/apl34.1.2016.06

Divaris, K., Lai, C. S., Polychronopoulou, A., Eliades, T., y Katsaros, C. (2012). Stress and burnout among swiss dental residents. Shweiz Monatsschr Zahmed, 122(7-8), 610-615.

Ferrer, A., Solano, M., Vizcaya, M., y Vidal, P. (2002). Síndrome de burnout en profesionales de enfermería de cuidados críticos. Enfermería Intensiva, 13(1), 9-16. Descargado de https://doi.org/10 .1016/S11302399(02) 78049-8

Freudenberger, H. (1974). Staff burn-out. Journal of Social Issues, 30(1), 159-165. Descarga- do de https://doi.org/10.1111/j.1540 -4560.1974. tb00706.x

Greig, D., y Fuentes, X. (2009). "burnout” o agotamiento en la práctica diaria de la medicina. Revista Chilena de Cardiología, 28(4), 403-407. Descargado de https://doi.org/10.4067/s0718 $-85602009000300013$

Hernández Sampieri, R., Fernández Collado, C., y Baptista Lucio, P. (2010). Metodología de la investigación. México D.F.: Editorial McGraw-Hill.

LaPorta, L. D. (2010). Occupational stress in oral and maxillofacial surgeons: Tendencies, traits, and triggers. Oral and Maxillofacial Surgery Clinics of North, 22(4), 495-502. doi: 10.1016/j.coms.2010.07.006

López, F., y Bernal, L. (2002). Prevalencia y factores asociados con el síndrome de burnout en enfermería de atención hospitalaria. Revista de Calidad Asistencial, 17(4), 201-205. Descargado de https://doi . org/10.1016/S1134282X (02) 77505-2

Marenco-Escuderos, A., y Ávila Toscano, J. (2016). Burnout y problemas de salud mental en docentes: diferencias según características demográficas y sociolaborales. Psychologia: Avances de la Disciplina, 10(1), 91-100.

Martínez, N., Vilaregut, A., y Roig, A. (2004). Prevalencia del síndrome de burnout y el estado general de salud en un grupo de enfermeras de un hospital de barcelona. Enfermería Clínica, 14(3), 142- 151. Descargado de https://doi.org/10.1016/ S1130-8621 (04) 73873-5

Maslach, C., Jackson, S., Leiter, M., Schaufeli, W., y Schwab, R. (1986). Maslach burnout inventory. Palo Alto: CA: Consulting Psychologists Press.

Navarro-Abal, Y., Climent-Rodríguez, J., y López-López, M. (2017). Ansiedad, gestión conductual del conflicto y estrategias de afrontamiento en profesionales de la orientación laboral. Universitas Psychologica, 16(4), 1-8. Descargado de https://doi.org/ 10.11144/Javeriana.upsy16-4.agcc

Ortega, C., y López, F. (2004). El burnout o síndrome de estar quemado en los profesionales sanitarios: revisión y perspectivas. International Journal of Clinical and Health Psychology, 4(1), 137-160.

Pozos, B. E., Torres, T. M., Aguilera, M. A., Acosta, M., y González, G. J. (2008). Stress-associated factors in mexican dentists. Brazilian Oral Research, 22(3), 223-228.

Pozos, B. E., Zaragoza, S., Aguilera, M. A., Acosta, M., Torres, T. M., y Ramírez, M. A. (2011). El cirujano dentista ante el estrés no percibido como un riesgo ocupacional. Revista Cubana de Salud y Trabajo, 12(1), 10-15.

Rick, J., Briner, R., Daniels, K., Perryman, S., y Guppy, A. (2001). A critical review of psychosocial hazard measures. HSE Books.

Rolander, B., Stenstrom, U., y Jonker, E. (2008). Relations- 
hip between psychosocial work environment,factors, personality, physical work demands and workload in a group of swedish dentists. Swedish Dental Journal, 32(4), 197-203.

Salgado-Roa, J. A., y Lería-Dulčić, F. (2020). Burnout, satisfacción y calidad de vida laboral en funcionarios de la salud pública chilenos. Univ. Salud., 22(1), 616. doi: https://doi.org/10.22267/rus.202201.169

Recibido: 13 de enero de 2021

Aceptado: 8 de marzo de 2021

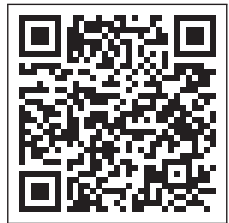

